\title{
The effectiveness of virtual reality and treadmill training in Parkinson's disease patients
}

\begin{abstract}
Background: Parkinson's disease (PD) in a neurodegenerative disorder that is mainly managed with medication and conventional physiotherapy. Virtual reality (VR) and treadmill training (TT) can be used in rehabilitation programs for PD patients to improve their motor control, balance, functional mobility and gait problems.

Objective: The aim of this review was to collect the current evidence on these interventions and assess their effectiveness on patients with Parkinson's disease.

Methodology: After electronic searches through databases and search engines, 19 articles were identified as suitable for the review focusing on a total of 952 PD patients participating in either VR or TT interventions.

Main Results: The VR intervention significantly improved balance, gait, daily life activities and quality of life of PD patients compared to a passive control group and increased avails on stride and step length compared with an active control group ( $p$-value $<0.05)$. Similarly, the TT intervention was shown to significantly improve gait speed and balance performance of PD patients compared to passive control group and gait speed, stride length and walking distance compared with an active control group ( $\mathrm{p}$-value $<0.05$ ).

Conclusion: Both VR and TT approaches have beneficial effects on PD patients and the interventions should further explored, enhanced and applied to improve the rehabilitation process of the patients.
\end{abstract}

Keywords: Parkinson's disease, virtual reality, treadmill training
Volume 10 Issue 4 - 2018

\author{
A Kottas Loizos,' G Gkrimas, ${ }^{2}$ M Pyrgeli \\ 'Physiotherapist, Greece \\ ${ }^{2}$ Biomechanist, Head of Gait \& Motion Analysis Center, Greece \\ ${ }^{3}$ Doctor of Physical \& Rehabilitation Medicine, Scientific \\ Director of ELEPAPAthens, Greece
}

Correspondence: M Pyrgeli, Doctor of Physical \&

Rehabilitation Medicine, Scientific Director of ELEPAP Athens,

Greece, Email mariapyrdoc@yahoo.gr

Received: July 12, 2018 | Published: July 24, 2018
Abbreviations: PD, Parkinson's disease; VR, virtual reality; TT, treadmill training; BWS, bodyweight support; RCT, randomized control trial; BBS, berg balance scale; LOS, limits of stability; FGA, functional gait assessment; TUG, timed up and go; SLS, single leg stance; RST, rapid-step up test; MCT, Motor Control Test; SOT, sensory organization test; $\mathrm{ABC}$, activities-specific balance confidence; MMSE, mini-mental scale examination; MCA, montreal cognitive assessment

\section{Introduction}

Parkinson's disease (PD), was described by James Parkinson and it is the second most common progressive neurodegenerative disorder after Alzheimer's disease. ${ }^{1}$ It is characterized by the degeneration of dopaminergic neurons within the substantia nigra, which leads to dopamine loss in the striatum and subsequently to motor deficits. By the time of death, approximately $50-70 \%$ of the neurons in this region of the brain have been lost compared to healthy individuals. ${ }^{2}$

It is estimated that there are approximately 10 million PD patients worldwide (less than $1 \%$ of whole population), but generally the epidemiology data are limited in many countries. ${ }^{3}$ A systematic review. ${ }^{4}$ which included 15 countries and individuals over 50years old, showed 4.1-4.6million people living with the disease. These numbers are expected to increase to 8.7-9.3millions until 2030. In the United States of America there are $c a$. 1million PD patients. ${ }^{3}$ in UK approximately $130.000,{ }^{5}$ in Germany $c a .275 .000^{6}$ while Western Europe has prevalence $160 / 100.000^{3}$ and China $190 / 100.000$ people. ${ }^{7}$ PD usually appears at the age of 55-75. At younger ages (40-49) the prevalence is $41 / 100.000$ and it gradually increases until it reaches $1.903 / 100.000$ individuals over the age of 80 . Regarding gender, there is a slight preponderance of evidence in male population at the majority of the age groups. ${ }^{8}$ The cardinal signs of PD are related to motor problems, grouped under the acronym TRAP, and include Tremor, Rigidity, Akinesia and Postural instability. Resting Tremor is decreasing on action and is the most common symptom affecting mainly the hands but also chin, jaw and lips. Rigidity is increased resistance and the existence of the 'cogwheel' phenomenon. Akinesia (bradykinesia) refers to the slowness of voluntary movements and reaction times and is also associated with difficulties in planning and performing simultaneous tasks. Postural instabilityis accompanied by an increased risk of falling. ${ }^{1,3,9,10}$ Gait impairment, which is interdependent with postural stability, is also frequent as PD progresses, including decreased walking speed, stride and step length, increased cadence and freezing of gait (i.e. inability to initiate gait by producing effective steps or complete stepping cessation during gait). ${ }^{11}$ Additionally, non-motor symptoms may be observed such as cognitive impairment, dementia ( $\sim 30 \%$ of patients), depression ( $\sim 40 \%$ of patients), other sensory symptoms (paresthesia, anosmia), dysautonomia (orthostatic hypotension, sexual dysfunction) and sleep disorders. ${ }^{1,3,9,12}$

Virtual reality (VR) technology can be used as a new rehabilitation tool in a variety of conditions and with different ways of application in order to achieve optimal motor learning and cognitive practice, in a safe environment, by providing feedback. ${ }^{13}$ VR is recommended for practicing new motor strategies and abilities that have been lost due to a disease or an injury. VR, by providing the opportunity to the individual to replicate scenarios from their daily life, challenges and motivates them and thus increases the possibilities for functional activities in real life. ${ }^{14}$

Treadmill training (TT) is a new electromechanical device that can be used in Parkinson's patients' rehabilitation program and more 
generally in hemiparetic individuals. ${ }^{15}$ TT focuses mainly on gait problems, such as gait speed and sufficient step length, and has been found to improve these parameters. ${ }^{10}$ Additionally, if the patient faces strength and balance problems there is the option for bodyweight support (BWS) during TT to facilitate them during the procedure. ${ }^{15}$ Since the effectiveness of VR and TT on PD patients is not clear but potential advantages exist, a literature review is required to summarize the benefits of each intervention. Therefore, the aim of this review is to summarize and assess the effectiveness of VR and TT as treatments on patients with Parkinson's disease.

\section{Methodology}

A computerized literature search of electronic databases was performed on Cochrane, PEDro, Pubmed and Science Direct by two researchers independently, using the keywords: Parkinson AND virtual reality; Parkinson AND treadmill training. In total 677 relevant articles were identified and initial screening excluded 568 of them because they did not meet the predefined inclusion criteria as described below. Two additional articles were identified from crosschecking of references, citations in review papers.

The inclusion criteria are stated below: (1) the article needed to be a Randomized Control Trial (RCT); (2) the article needed to have been published from 2007 until 2017; (3) the patients should have been diagnosed with PD; (4) the interventions could be either VR or TT; (5) the adults needed to be 55 year or older and (6) the article needed to be in English. Following full-text accession of the remaining 109 articles; 27 were included in this review together with the two articles from additional sources (Figure 1). Before proceeding, the quality of the articles was assessed using the PEDro scale and all of them were found to be of high (PEDro score 6-10) or fair (PEDro score 4-5) quality (Tables 1) (Table 2).

\section{Sample characteristics}

A total of $952 \mathrm{PD}$ patients were included, 560male (58.8\%) and 392 female $(41.2 \%)$, with a mean age of $67.05( \pm 7.74)$ years. The majority of the trials had small sample sizes:24 trials involved fewer than 50 participants ${ }^{16-39}$ with 11 out of them involving fewer than 25 PD patients ${ }^{17,22,27-29,32,35-37,39,40}$ while only 5 trials had over 50 participants. ${ }^{10,41-44}$

All studies except one ${ }^{32}$ clearly specified inclusion and exclusion criteria. The stage of the disease varies as 4 studies included participants in the early stages, ${ }^{16,22,24,30} 12$ studies early to moderate, ${ }^{10,17,21,25,26,28,29,33,34,38,39,42} 6$ studies mild to moderate, ${ }^{19,20,23,31,36,44}$ 3 studies moderate, ${ }^{18,37,41} 2$ studies mild to severe, ${ }^{27,35}$ and 1 study included participants from all the stages of the disease..$^{43}$ Participants that were medically unstable, suffered from another neurological condition apart from PD, orthopedic issues, cardiopulmonary problems, visual or audial deficits, cognitive impairment (MMSE $<24$ ), dementia, depression or had history of Deep Brain Stimulation (DBS) were excluded from all trials.

\section{Interventions}

The main focus of all trials was motor rehabilitation, with 5trials focusing exclusively on the improvement of balance performance, 9 focusing exclusively on the improvement of gait performance (spatiotemporal parameters) and 15 on both. Additionally, the total dose of therapy varied among studies, ranging from 6hours to 72 hours, at a frequency of $2-7$ times/week and spread over a total period 4-24weeks. Finally, outcome measures were collected at baseline and post-intervention. The follow-up period differs, as for most trials it was $1-4$ months and only two trials reported a follow-up after the longer period 6 and 12 months.

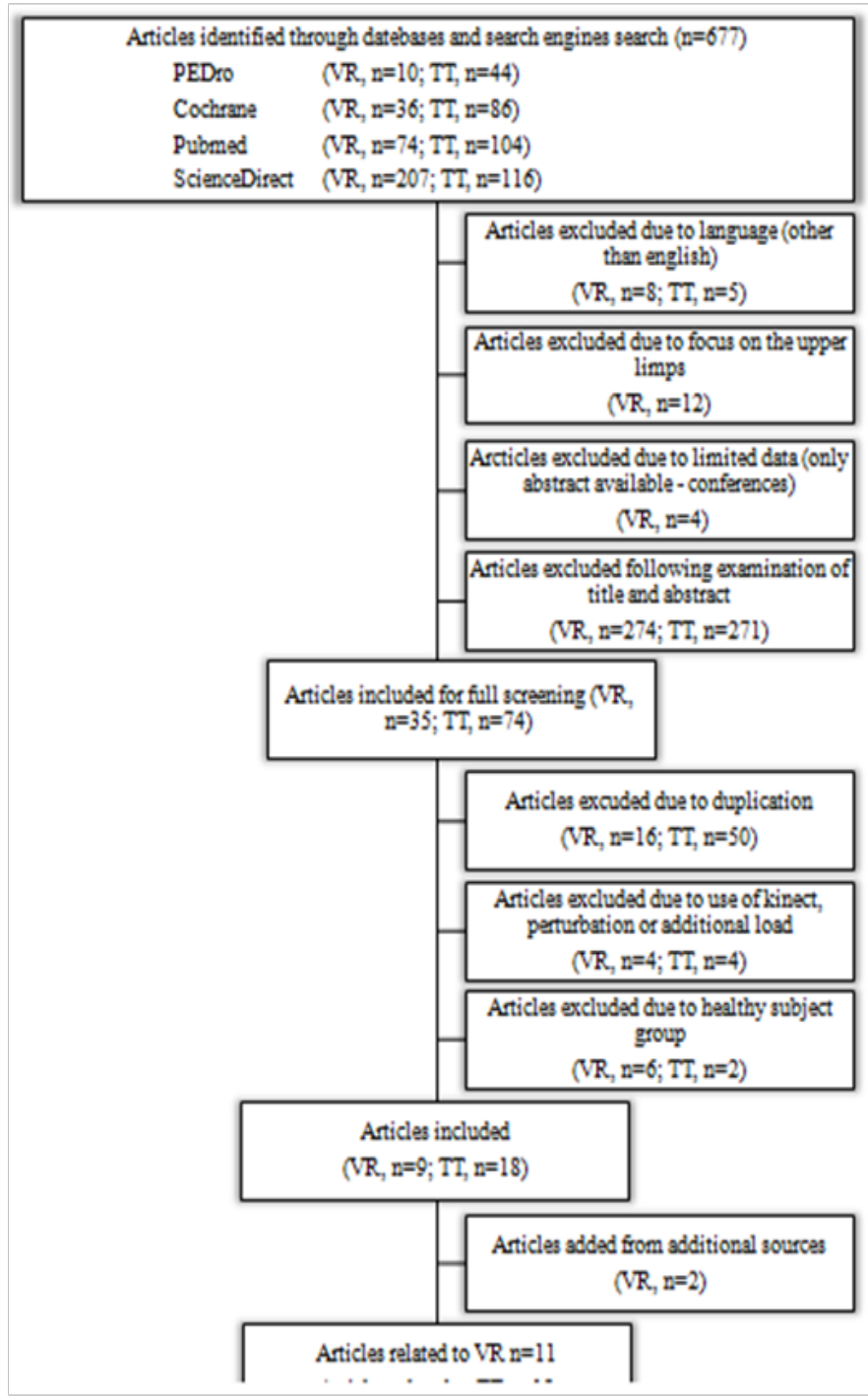

Figure I

\section{Discussion}

The main findings of studies applying VR on PD patients are: 1) significant improvement in balance, gait, daily life activities and quality of life compared to a passive control group; 2) increased avails on stride and step length compared with an active control group; 3) similar effects on balance, ADL activities, gait, cognitive function and quality of life compared with an active intervention group (Table 1).

The main findings of studies applying TT on PD patients are: 1) significant improvement of gait speed and balance performance compared to passive control group; 2) significant benefits on gait speed, stride length and walking distance compared with an active control group; 3) similar effects on balance performance, step length, step width and cadence compared with an active intervention group (Table 2). 


\section{Primary outcomes}

\section{Gait}

The short-term effect of VR treatment on gait compared to a passive control group was assessed with the use of GAITRite walkway and Functional Gait Assessment that was measuring stride velocity and length during obstacle crossing or without obstacles and walking ability (FGA). For all characteristics, there was a significant improvement in the VR group compared to the passive control group (obstacle crossing stride velocity and length $\mathrm{p}<0.01$; FGA, stride velocity and length $\mathrm{p}<0.05) .{ }^{33,34}$ No long-term comparison was included.

The short-term effect of TT on gait compared to a passive control group was assessed with the use of 10-meter walk test, 6-minute walk test, Dynamic Gait Index, GAITRite or 3D kinematic analysis that was measuring speed, stride-step length, step width, distance and cadence. Speed was found significantly improved $(p<0.05)$, similarly to stride length, step length and distance ( $\mathrm{p}<0.01, \mathrm{p}<0.01 \& \mathrm{p}<0.05$ respectively); step width and cadence showed no improvement in the TT group compared to passive control group..$^{10,16,17.22,30,37}$ No long-term comparison was included.

The short-term effect of VR treatment on gait compared to an active control group was assessed with the use of GAITRite walkway, Dynamic Gait Index, FGA, 10m walk test and 6-minute walk test that was measuring stride velocity and length during obstacle crossing or without obstacles and walking ability (FGA). Gait performance and speed were significantly improved in both VR and active control group, while step and stride length showed increased improvement in the VR group. . $^{31,33,34,36,39,43,44}$ The long-term effect of VR on gait with an active control group was assessed with GAITRite walkway that measured gait velocity and stride length 3 months and 12 months after treatment. In both follow-ups gait velocity was improved equally in both VR and active control, while stride length was improved only in the VR group. ${ }^{43}$

The short-term of TT on gait compared to an active control group was assessed with the use of 10-meter walk test, 6-minute walk test, GAITRite, 3D kinematic analysis or the training treadmill was measuring gait speed, stride-step length, step width, cadence and distance. Gait speed, stride length and distance were significantly improved in TT groups $(\mathrm{p}<0.05)$, while step length, step width and cadence were equally improved in both TT and active control group. Additionally, robotic gait intervention demonstrated similar improvements with TT, high intensity TT showed fewer benefits in comparison with intermediate and low intensity, while the combination on TT with visual or/and audial cues had improved benefits. ${ }^{16,18,19,21,23,25,28-30,35,38,41,42}$ The long-term effects of TT (6months) in comparison to an active control group was assessed with the use of 10-meter walk test and 10-minute walk test and showed lasting positive effects on gait speed and walking distance $(\mathrm{p}<0.05 \&$ $\mathrm{p}<0.001){ }^{21}$

\section{Balance}

The short-term effect of VR treatment on balance performance compared to a passive control group was assessed with the use of Timed Up and Go, Berg Balance test and Sensory Organization Ability (indirectly) and the results showed the significant benefits of VR intervention (TUG $\mathrm{p}<0.01$; BBS, vision and vestibular $\mathrm{p}<0.05$ ). ${ }^{32-34}$ The short-term effect of TT on balance compared to a passive control group was assessed with the use of Berg Balance Scale (BBS), Timed Up and Go (TUG) and Activities-specific Balance Confidence (ABC) and the results demonstrated significant improvements on balance performance compared to a passive control group $(\mathrm{p}<0.05) .{ }^{10,17,30}$

The short-term effect of VR treatment on balance performance compared to an active control group was assessed with the use of Berg Balance Scale, Timed Up and Go, Single Leg Stance, Limits of Stability, Activities-Specific Balance Confidence and Sensory Organization Ability (indirectly) and the results demonstrated equally significant improvements in both groups ${ }^{24,31,33,34,36,43,44}$ solely Ribas et al. ${ }^{39}$ showed a superior effect of VR on balance. The long-term effect of VR on balance with an active control group was assessed by ABC scale and SLS and balance was measured on 3months and 12months follow-up. The results showed significant improvement in favor of VR in ABC scale after both 3 and 12months, while SLS did not show any improvements. ${ }^{43}$

The short term effect of TT on balance performance compared to an active control group was assessed with the use of Berg Balance Scale (BBS), Limits Of Stability (LOS), Functional Gait Assessment (FGA), Timed Up and Go (TUG), Single Leg Stance (SLS), Rapid-Step up Test (RST), Motor Control Test (MCT) and Sensory Organization Test (SOT) and Activities-specific Balance Confidence (ABC) and the results demonstrated similar to superior results in favor of the TT group. ${ }^{19,21,25,28,29,35,38,41}$ The investigation of the long-term effects of TT compared to an active control group showed significant improvement in favor of TT (TUG p<0.01) in 6-months follow-up. ${ }^{21}$

\section{Secondary outcomes}

\section{ADL-QoL-muscle strength (VR vs passive control group)}

The secondary outcomes of VR intervention compared to a passive control group were assessed by Lee et al. ${ }^{32}$ with the use of Modified Barthel Index and the results showed significant differences in the ADL in favor of VR $(p<0.05)$. Liao et al. ${ }^{33}$ measured quality of life with the use of PDQ-39 and showed significant benefits to the VR group compared to a passive control group $(\mathrm{p}<0.01)$. Additionally, a significant improvement was found regarding strength $(\mathrm{p}<0.05)$ by Liao et al. ${ }^{34}$ using a dynamometer.

\section{Global motor function-cognition-fatigue (TT vs passive control group)}

The secondary outcomes of TT compared to a passive control group were assessed by Canning et al., ${ }^{22}$ Nadeau et al., ${ }^{30}$ Picelli et al. ${ }^{37}$ on global function with the use of UPDRS and only the latter found significant results $(\mathrm{p}=0.013)$ in favor of TT, while the others showed similar effect. Cognition was assessed by the use of Mini-Mental Scale Examination (MMSE) and Montreal Cognitive Assessment (MCA) with improvement on one of the measures $(\mathrm{p}=0.012)$, while fatigue was measured by Canning et al. ${ }^{22}$ and was found significantly improved $(\mathrm{p}<0.05)$ by the use of Visual Analogue Scale to Evaluate Fatigue Severity (VAS-F).

\section{Depression-QoL-falls (TT vs passive control group)}

Assessment of depression and falls between TT and a passive control group showed a positive trend via Beck Depression Index (BDI), ${ }^{30,37}$ while Cakit et al. ${ }^{10}$ demonstrated improvement regarding falls with the use of Falls Efficacy Scale (FES) $(p<0.01)$. No differences were found between the groups on the measurement of quality of life with PDQ-39. 


\section{Global motor function-fatigue (VR vs active control group)}

UPDRS-III and Fatigue Severity Scale (FSS) were used for the assessment of global motor function and fatigue between VR treatment and an active control group. Van den Heuvel et al. ${ }^{31}$ and Ribas et al. ${ }^{39}$ found significant benefits in favor of VR interventions in UPDRS-III and FSS respectively, while Pompeu et al. ${ }^{24}$ found no significant differences between interventions.

\section{Quality of life (VR vs active control group)}

Quality of life was measured by the use of PDQ-3926,31,33,36 or PDQ$8 .{ }^{44}$ In the majority of trials, the results showed similar improvement; only Pedeira et al. ${ }^{26}$ reported that the benefit was greater for the VR intervention.

\section{ADL-Cognitive function-muscle strength (VR vs active control group)}

Measurements of ADL with UPDRS-II, ${ }^{24}$ cognitive function with Montreal Cognitive Assessment ${ }^{24}$ and muscle strength with a dynamometer ${ }^{34}$ indicating significant improvements in both VR group and active control group.

\section{Global motor function-fatigue-falls-QoL (TT vs active control group)}

Global motor function was measured with UPDRS and the results are similar or superior in favor of the TT group. ${ }^{18,21,25,30,35,41}$ Fatigue was found significantly improved $(\mathrm{p}=0.001)$ in the Parkinson's Fatigue Scale (PFS) ${ }^{38}$ and falls were also improved as shown by the FES $(\mathrm{p}<0.001)^{38}$ and SOT-Falls $(\mathrm{p}=0.045) .{ }^{29} \mathrm{QoL}$ showed similar results in Nadeau et al. ${ }^{30}$ and Carda et al. ${ }^{10}$ and only in one trial ${ }^{29}$ there were significant results in PDQ-39 ( $\mathrm{p}=0.016)$.

\section{Cognition-depression-muscle strength (TT vs active control group)}

No significant results were noted between the groups on the assessment of cognition with MMSE, depression with $\mathrm{BDI}^{30}$ and of muscle strength with 1-Repetition Maximum. ${ }^{42}$ The rationale of using VR and TT is to provide external cues, such as visual, auditory, somatosensory and proprioceptive feedback, and cognitive stimulation while in the same time presenting a motivating stimulus, which together with the repetitive nature of the games are facilitating motor relearning and promote musculoskeletal disorders' rehabilitation. ${ }^{45-47}$

In VR, by watching the movements of their avatar and receiving the appropriate feedback, patients can challenge their postural control, strengthen the stimulation of proprioceptors and the vestibular system and possibly bypass their motor deficiency and improve motor response. ${ }^{48,49}$ So VR is enabling the increased attention and focus on mobility in order to perform in the game and the exploitation of the motor muscle system, cerebellum and oculomotor. ${ }^{47}$ TT, without the combination of visual or audial cues, is beneficial for postural control and the continuous stimulation provided acts as an external pacemaker, improving body alignment and walking rhythm. ${ }^{50,51}$ In combination with audial or/and visual cues the external rhythm benefits even more, since the signals can be used to trigger intact pathways and bypass pallidocortical circuit to facilitate movement. ${ }^{40}$ The visual feedback can create modifications in the optical flow that lead to modification in the gain pattern ${ }^{52}$ and the stimulus can be used as an anchor for the patient to maintain a stable position and focus on the task. ${ }^{53}$ Additionally, the cursive speed maintain by the treadmill can restrict the patients and help in the normalization of stride-tostride gait pattern. ${ }^{54}$ Physical exercise by the use of video games or treadmill is beneficial as the intensity of training and feedback can be tailored in order to create the most suitable, individualized therapeutic protocol to facilitate motor learning. ${ }^{55}$ The high number of repetitions of target-movements may also favor learning and, in extent, lead to changes of brains' architecture..$^{39}$ TT may be a way to apply many gait cycle repetitions to get results, even though it may be considered a forced-use-therapy as the patient is forced to walk faster than he would choose if he was over ground. ${ }^{56}$ This forced therapy may lead to changes in central motor control processes due to increases in cortical and subcortical activation. ${ }^{57}$

Patients with impaired basal ganglia might learn to integrate vestibular, visual and somatosensory information through the cerebellum, which would influence the brain stem and spinal cord to improve postural control. Basal ganglia are important to maximize the reward and internal feedback, while cerebellum was suggested to be responsible for supervised learning and feedforward. Additionally, cerebellum is important to assist in postural control by executing the vestibular spinal reflex, after integrating multiple sensory information from audial, visual and somatosensory components. ${ }^{58}$ The similar results between VR or TT and conventional physiotherapy can be attributed to the nature of the exercises. The programs were using the same motor demands and so there were leading in similar training effects. $^{59}$

VR exercise demonstrated increased benefits for step and stride length and TT in gait speed and stride length, whose decrease is a main symptom of PD also linked to increased gait variability and doublestance time. ${ }^{60}$ Gait patterns may still be generated normally while a reduction in automaticity is observed, therefore there is a need to bypass automatic control mechanisms. ${ }^{61}$ The observed improvement may be attributed to the fact that VR and TT provides a more accurate and complete motor feedback and so amplitude stride correction is better that in convention physiotherapy. Postural instability in PD is considered one of the most incapacitating motor symptoms that dopaminergic therapy does not significantly improve. ${ }^{62}$ According to Schoneburg et al. ${ }^{63}$ there are four control systems involved in managing balance: 1) static balance; 2) reactive postural adjustments; 3) anticipatory postural adjustments; 4) dynamic balance. In patients with PD, all these systems are possibly affected and it is not clear whether VR or TT exert their positive effects by improving general balance or selectively influencing specific postural control systems.

Balance performance is mainly estimated by Berg Balance Scale which is a robust measure, ${ }^{64}$ but is also characterized by substantial ceiling and floor effects. ${ }^{65}$ The use of more sensitive tools, such as objective posturography techniques ${ }^{66}$ or the novel clinical test MiniBESTest ${ }^{67}$ can reveal smaller balance alternations, uncover balance improvements in PD patients and clarify the degree of effectiveness of VR-based exercises and TT in future studies. Barry et al. ${ }^{68}$ suggested that VR rehabilitation has some drawbacks, such as cyber-sickness, inappropriate level and/or content of exercises and cognitive overload. All can be overcome with the use of customized, PD-specific VR applications, which could even be proven superior to commercial VR systems.

The possibility of a home-based VR system or TT for exercise is a great advantage, even though safety issues may occur, as it will add flexibility on patient rehabilitation and may increase the exercise dependence for patients that tend to drop out. ${ }^{69}$ For this application 
it is important to investigate whether the existence of minimum supervision can yield the same quality of treatment. ${ }^{70}$ Furthermore, the home-based VR treatment should ensure that game performance will not depend on compensatory movements, so patients will not prioritize game scores over improvement of quality of movement, since gaining points and achieving higher scores provide increasing motivation for practice.

\section{Limitations}

With the exception of of Cakit et al,. ${ }^{10}$ Picelli et al,. ${ }^{41}$ Shulman et al, ${ }^{42}$ Shen \& Mak, ${ }^{43}$ Gandolfi et al,,${ }^{44}$ all others trials had small sample sizes reflecting the low certainty in the generalization of the outcomes of interest. Randomization was performed in the vast majority of the studies, ${ }^{32}$ while allocation concealment in almost half of the studies. . $^{10,16-19,23,25,28-30,32,38,41,42,44}$ Blinding of the outcome assessors was performed in almost all the studies ${ }^{18,25,32,35}$ while blinding of the subjects and therapists was not applicable due to the nature of the VR and treadmill interventions. Almost $1 / 3$ of the studies that incurred subject losses did not perform an intention-to-treat analysis; Liao et al, ${ }^{33}$ Liao et al,. ${ }^{34}$ Harro et al,. ${ }^{28}$ Harro et al,. ${ }^{29}$ had limited dropout (1 subject), Shulman et al..$^{42}$ had $16 \%$ drop-out, Nadeau et al. ${ }^{30}$ had $24 \%$, Pedreira et al. ${ }^{26}$ had $27 \%$, Schlick et al. ${ }^{35}$ had $35 \%$ and Cakit et al. ${ }^{10}$ had $43 \%$.

Additional high-quality studies with larger sample sizes should be performed to investigate more extensively the potential benefits of VR and TT as also the mechanism of its influence on PD patients. The standardization of the measured outcomes together with the training intensity, frequency and duration is necessary, since consolidation period is necessary for motor learning. ${ }^{71}$ Since high intensity of TT might not be required to achieve significant improvements, later stages of PD patients can benefit from lower intensity training. Furthermore, longer follow-up periods should be applied to investigate long-term effects of VR and TT. Also both should be investigated at different stages of the disease in order to identify opportunities to delay PD progress in early stages or understand whether it should be applied with caution in later stages because of the cognitive impairment. Additionally, testing the interventions during both the 'on' and 'off' periods of PD medication is necessary to eliminate its confounding effects. Differences between customized and commercial VR need to be defined to differentiate if one is more suitable than the other.

Finally, the combination of TT and VR is proposed to improve gait parameters and balance in PD patients. Mirelman et al. ${ }^{72}$ has already showed benefits through this combination, in gait speed, stride length and cognition, while there are also demonstrated benefits in functional gait training in children with cerebral palsy ${ }^{73}$ and improvements in gait and balance in early stroke patients..$^{74,75}$

\section{Conclusion}

Both VR and TT have shown positive results in the rehabilitation of PD. Both can improve gait parameters as walking speed and stride length and also balance seems to be affected positively. The mechanism and the degree of affection is not yet clear, so there is a need for further research with larger sample and standardized parameters to fully integrate VR and TT into the rehabilitation of PD patients.

\section{Acknowledgements}

None.

\section{Conflict of interest}

The author declares that there is no conflict of interest.

\section{References}

1. Wirdefeldt K, Adami HO, Cole P, et al. Epidemiology and etiology of Parkinson's disease: o review of the evidence. Eur J Epidemiol. 2011;26(1 suppl):1-58S.

2. Davie CA. A review of Parkinson's disease. Br Med Bull. 2008;86(1):109127.

3. APDA. What is Parkinson's disease. Understanding the basics of Parkinson's disease. 2017.

4. Dorsey ER, Constantinescu R, Thompson JP, et al. Projected number of people with Parkinson disease in the most populous nations, 2005 through 2030. Neurology. 2007;68(5):384-386.

5. NHS. Parkinson's disease. Overview; 2016.

6. Nerius M, Fink A, Doblhammer G. Parkinson's disease in Germany: prevalence and incidence based on health claims data. Acta Neurol Scand. 2017;136(5):386-392.

7. Ma CL, Su L, Xie JJ, et al. The prevalence and incidence of Parkinson's disease in China: a systematic review and meta-analysis. J Neural Transm (Vienna). 2014;121(2):123-134.

8. Prinqsheim T, Jette N, Frolkis A, et al. The prevalence of Parkinson's disease: a systematic review and meta-analysis. Mov Disord. 2014;29(13):1583-1590.

9. Jankovic J. Parkinson's disease: clinical features and diagnosis. Journal of Neurology, Neurosurgery and Psychiatry. 2008;79(4):368-376.

10. Cakit BD, Saracoglu M, Genc H, et al. The effects of incremental speeddependent treadmill training on postural instability and fear of falling in Parkinson's disease. Clin Rehabil. 2007;21(8):698-705.

11. Earhart GM, Williams AJ. Treadmill training for individuals with Parkinson disease. Phys Ther. 2012;92(7):893-897.

12. Wajda DA, Mirelman A, Hausdorff JM. et al. Intervention modalities for targeting cognitive-motor interference in individuals with neurodegenerative disease: a systematic review. Expert Rev Neurother. 2017;17(3):251-261.

13. Dockx K, Bekkers EM, Van der berqh V, et al. Virtual reality for rehabilitation in Parkinson's disease. Cochrane Database Syst Rev. 2016;12:CD010760..

14. Goble DJ, Cone BL, Fling BW. Using the Wii Fit as a tool for balance assessment and neurorehabilitation: the first half decade of "Wii-search". J Neuroeng Rehabil. 2014;11:12.

15. Mehrholz J, Pohl M, Elsner B. Treadmill training and body weight support for walking after stroke. Cochrane Database Syst Rev. 2014;23(1):CD002840.

16. Fisher BE, WU AD, Salem GJ, et al. The effect of exercise training in improving motor performance and corticomotor excitability in people with early Parkinson's disease. Arch Phys Med Rehabil. 2008;89(7):12211229.

17. Kurtais Y, Kutlay S, Tur BS, et al. Does treadmill training improve lowerextremity tasks in Parkinson disease? A randomized controlled trial. Clin J Sport Med. 2008;18(3):289-291.

18. Frazzitta G, Maestri R, Uccellini D, et al. Rehabilitation treatment of gait in patients with Parkinson's disease with freezing: a comparison between two physical therapy protocols using visual and auditory cues with or without treadmill training. Mov Disord. 2009;24(8):1139-1143.

19. Chaiwanichsiri D, Wangno W, Kitisomprayoonkul W. et al. Treadmill 
training with music cueing: a new approach for Parkinson's gait facilitation. Asian Biomedicine. 2011;5(5):649-654.

20. Yen $\mathrm{CY}$, Lin $\mathrm{KH}, \mathrm{Hu} \mathrm{MH}$, et al. Effects of virtual-augmented balance training on sensory organization and attentional demand for postural control in people with Parkinson disease: A randomized controlled trial. Phys Ther. 2011;91(6):862-874.

21. Carda S, Invernizzi M, Baricich $\mathrm{A}$, et al. Robotic gait training is not superior to conventional treadmill training in Parkinson disease: a single-blinded randomized controlled trial. Neurorehabil Neural Repair. 2012;26(9):1027-1034

22. Canning CG, Allen NE, Dean CM, et al. Home-based treadmill training for individuals with Parkinson's disease: a randomized controlled pilot trial. Clin Rehabil. 2012;26(9):817-826.

23. El-Tamawy MS, Darwish MH, Khallaf ME. Effects of augmented proprioceptive cues on the parameters of gait of individuals with Parkinson's disease. Ann Indian Acad Neurol. 2012;15(4):267-272.

24. Pompeu JE, Mendes FA, Silva KG, et al. Effect of Nintendo Wii -based motor and cognitive training on activities of daily living in patients with Parkinson's disease: a randomized clinical trial. Physiotherapy. 2012;98(3):196-204.

25. Bello O, Sanchez JA, Lopez-alonso V, et al. The effect of treadmill or overground walking training program on gait in Parkinson's disease. Gait Posture. 2013;38(4):590-595.

26. Pedreira G, Prazeres A, Cruz D, et al. Virtual games and quality of life in Parkinson's disease: A randomized controlled trial. Advances in Parkinson Disease. 2013;2(4):97-101.

27. Sale $\mathrm{P}$, de pandis MF, Le pera $\mathrm{D}$, et al. Robot-assisted walking training for individuals with Parkinson's disease: a pilot randomized controlled trial BMC Neurol. 2013;13:50.

28. Harro CC, Shoemaker MJ, Frey OJ, et al. The effect of speed-dependen treadmill training and rhythmic auditory-cued overground walking on gai function and fall risk in individuals with idiopathic Parkinson's disease: a randomized controlled trial. Neurorehabilitation. 2014;34(3):557-572.

29. Harro CC, Shoemaker MJ, Frey O, et al. The effects of speed-dependent treadmill training and rhythmic auditory-cued overground walking on balance function, fall incidence, and quality of life in individuals with idiopathic Parkinson's disease: a randomized controlled trial. Neurorehabilitation. 2014;34(3):541-556.

30. Nadeau A, Pourcher E, Corbeil P. Effect of 24 wk of treadmill training on gait performance in Parkinson's disease. Med Sci Sports Exerc. 2014;46(4):645-655

31. Van den Heuvel MR, Kwakkel G, Beek PJ, et al. Effects of augmented visual feedback during balance training in Parkinson's disease: a pilot randomized clinical trial. Parkinsonism Relat Disord. 2014;20(12):1352 1358.

32. Lee NY, Lee DK, Song HS. Effect of virtual reality dance exercise on the balance, activities of daily living and depressive disorder status of Parkinson's disease patients. J Phys Ther Sci. 2015;27(1):145-147.

33. Liao YY, Yang YR, Cheng SJ, et al. Virtual reality-based training to improve obstacle-crossing performance and dynamic balance in patients with Parkinson's disease. Neurorehabil Neural Repair. 2015;29(7):658667.

34. Liao YY, Yang YR, Wu YR, et al. Virtual reality-based Wii Fit training in improving muscle strength, sensory integration ability and walking abilities in patients with Parkinson's disease: A randomized controlled trial. International Journal of Gerontology. 2015;9(4):190-195.

35. Schlick C, Ernst A, Bötzel K, et al. Visual cues combined with treadmill training to improve gait performance in Parkinson's disease: a pilot randomized controlled trial. Clin Rehabil. 2016;30(5):463-471.

36. Yang WC, Wang HK, Wu RM, et al. Home-based virtual reality balance training and conventional balance training in Parkinson's disease: A randomized controlled trial. J Formos Med Assoc. 2016;115(9):734-743.

37. Picelli A, Varalta V, Melotti C, et al. Effects of treadmill training on cognitive and motor features of patients with mild to moderate Parkinson's disease: a pilot, single-blind, randomized controlled trial. Funct Neurol. 2016;31(1):25-31.

38. Pelosin E, Avanzino L, Barella R, et al. Treadmill training frequency influences walking improvement in subjects with Parkinson's disease: a randomized pilot study. Eur J Phys Rehabil Med. 2017;53(2):201-208.

39. Ribas CG, Alves DA, SilvaL, et al. Effectiveness of exergaming in improving functional balance, fatigue and quality of life in Parkinson's disease: A pilot randomized controlled trial. Parkinsonism Relat Disord. 2017;38:3-18.

40. Bello O, Fernandez-del-olmo M. How does the treadmill affect gait in Parkinson's disease? Current Aging Sci. 2012;5(1):28-34.

41. Picelli A, Melotti C, Origano F, et al. Robot-assisted gait training versus equal intensity treadmill training in patients with mild to moderate Parkinson's disease: a randomized controlled trial. Parkisonism Relat Disord. 2013;19(6):605-610.

42. Shulman LM, Katzel LI, Ivey FM, et al. Randomized clinical trial of 3 types of physical exercise for patients with Parkinson disease. JAMA Neurol. 2013;70(2):183-190.

43. Shen X, Mak MK. Balance and gait training with augmented feedback improves balance confidence in people with Parkinson's disease: A randomized controlled trial. Neurorehabil Neural Repair. 2014;28(6):524-535

44. Gandolfi M, Geroin C, Dimitrova E, et al. Virtual reality telerehabilitation for postural instability in Parkinson's Disease: A multicenter, singleblinded, randomized, controlled trial. BioMed Research Internation. 2017; ID 7962826:11

45. Rochester L, Baker K, Hetherrington V, et al. Evidence for motor learning in Parkinson's disease: acquisition, automaticity and retention of cued gait performance after training with external rhythmical cues. Brain Res. 2010;1319:103-111.

46. Leroi I, Andrews M, Mcdonald K, et al. Apathy and impulse control disorders in Parkinson's disease: a direct comparison. Parkinsonism Relat Disord. 2012;18(2):198-203.

47. Lee GH. Effects of virtual reality exercise program on balance, emotion and quality of life in patients with cognitive decline. Journal of Korean Physical Therapy. 2016;28(6):355-363.

48. Esculier JF, Vaudrin J, Bériault P, et al. Home-based balance training programme using Wii Fit with balance board for Parkinsons's disease: a pilot study. J Rehabil Med. 2012;44(2):144-150.

49. Mirelman A, Maidan I, Deutsch JE. Virtual reality and motor imagery: promising tools for assessment and therapy in Parkinson's disease. Mov Disord. 2013;28(11):1597-1608.

50. Herman T, Giladi N, Hausdorff JM. Treadmill training for the treatment of gait disturbances in people with Parkinson's disease: a mini-review. J Neural Transm (Vienna). 2009;116(3):307-318.

51. Trigueiro LC, Gama GL, Ribeiro TS, et al. Influence of treadmill gait training with additional load on motor function, postural instability and history of falls for individuals with Parkinson's disease: A randomized clinical trial. J Bodyw Mov Ther. 2017;21(1):93-100.

52. Ferrarin M, rabuffetti M, Tettamanti M, et al. Effect of optical flow versus attentional strategy on gait in Parkinson's Disease: a study with a portable 
optical stimulating device. J Neuro Eng Rehabil. 2008;5:3.

53. Dickstein R, Laufer Y. Light touch and center of mass stability during treadmill locomotion. Gait Posture. 2004;20(1):41-47.

54. Frenkel-Toledo S, Giladi N, Peretz C, et al. Treadmill walking as an external pacemaker to improve gait rhythm and stability in Parkinson's disease. Mov Disord. 2005;20(9):1109-1114.

55. de bruin ED, Schoene D, Pichierri G. et al. Use of virtual reality technique for the training of motor control in the elderly. Some theoretical considerations. Z Gerontol Geriatr. 2010;43(4):229-234.

56. Pohl M, rockstroh $\mathrm{G}$, Rückriem $\mathrm{S}$, et al. Immediate effects of speeddependent treadmill training on gait parameters in early Parkinson's disease. Arch Phys Med Rehabil. 2003;4(12):1760-1766.

57. Alberts JL, Linder SM, Penko AL, et al. It is not about the bike, it is about the pedaling: forced exercise and Parkinson's disease. Exercise and Sport Science Reviews. 2011;39(4);177-186.

58. Loffe ME, Chernikova LA, Ustinova KL. Role of cerebellum in learning postural tasks. Cerebellum. 2007;6(1):87-94.

59. Smania N, Corato E, Tinazzi M, et al. Effect of balance training on postural instability in patients with idiopathic Parkinson's disease. Neurorehabil Neural Repair. 2010;24(9):826-834.

60. Hausdorff JM. Gait dynamics in Parkinson's disease: common and distinct behavior among stride length, gait variability, and fractal-like scaling. Chaos. 2009;9(2): 026113.

61. Wu T, Hallett M, Chan P. Motor automaticity in Parkinson's disease. Neurobiol Dis. 2015;2:226-234.

62. Curtze C, Nutt JG, Carlson-kuhta P, et al. Levodopa is a double-edged sword for balance and gait in people with Parkinson's disease. Mov Disord. 2015;30(10):1361-1370.

63. Schoneburg B, Mancini M, Horak F, et al. Framework for understanding balance dysfunction in Parkinson's disease. Mov Dis. 2013;28(11):14741482 .

64. Steffen T, SENEY M. Test-retest reliability and minimal detectable change on balance and ambulation tests, the 36-item short-form health survey, and the unified Parkinson disease rating scale in people with parkinsonism. Phys Ther. 2008;88(6):733-746.
65. King LA, Priest KC, Salarian A, et al. Comparing the Mini-BESTest with the Berg Balance Scale to evaluate balance disorders in Parkinson's disease. Parkinson's Dis. 2012;2012:375419.

66. Nonnekes J, De kam D, Geurts AC, et al. Unraveling the mechanisms underlying postural instability in Parkinson's disease using dynamic posturography. Expert Rev Neurother. 2013;13(12):1303-1308.

67. Vervoort G, Bengevooed A, Nackaerts E, et al. Distal motor deficit contributions to postural instability and gait disorder in Parkinson's disease. Behav Brain Rev. 2015;287:1-7.

68. Barry G, Galna B, Rochester L. The role of exergaming in Parkinson's disease rehabilitation: a systematic review of the evidence. J NeuroEng and Rehabil. 2014;11:33.

69. Ellis T, Boudreau JK, de Angelis TR, et al. Barriers to exercise in people with Parkinson disease. Phys Ther. 2013;93(5):628-636.

70. King LA, Wilhelm J, Chen Y, et al. Effects of group, individual, and home exercise in persons with Parkinson disease: A randomized clinical trial. J Neurol Phys Ther. 2015;39(4):204-212.

71. Nieuwboer A, Rochester L, Muncks L, et al. Motor learning in Parkinson's disease: limitations and potential for rehabilitation. Parkinsonism Relat Disord. 2009;15(suppl 3):53-S58.

72. Mirelman A, Rochester L, Maidan I, et al. Addition of a non-immersive virtual reality component to treadmill training to reduce fall risk in older adults (V-TIME): a randomized controlled trial. Lancet. 2016;388(10050):1170-1182.

73. Van Gelder L, Booth ATC, Van de port I, et al. Real-time feedback to improve gait in children with cerebral palsy. Gait Posture. 2017;52:7682.

74. de rooij IJM, Van de port IGL, Meijer JWG. Feasibility and effectiveness of virtual reality training on balance and gait recovery early after stroke: A pilot study. International Journal of Physical Medicine \& Rehabilitation. 2017;5:418.

75. Mak MK, Pang MY, Mok V. Gait difficulty, postural instability, and muscle weakness are associated with fear of falling in people with Parkinson's disease. Parkinson's Dis. 2012;2012:901721. 